\title{
Do Community Characteristics Influence Unintended Pregnancies in Kenya?
}

\section{Bola Lukman Solanke ${ }^{1,3}$, Joseph Ayodeji Kupoluyi ${ }^{1,4}$,Joshua Odunayo Akinyemi ${ }^{2,3}$,Olufunmilayo Olufunmilola Banjo ${ }^{1,3}$}

\author{
1. Department of Demography and Social Statistics, Obafemi Awolowo University, Nigeria \\ 2. Department of Epidemiology and Medical Statistics, University of Ibadan, Nigeria \\ 3. Demography and Population Studies Programme, University of the Witwatersrand, South Africa \\ 4. Tropical Institute of Community Heath, Great Lakes University of Kisumu, Kenya
}

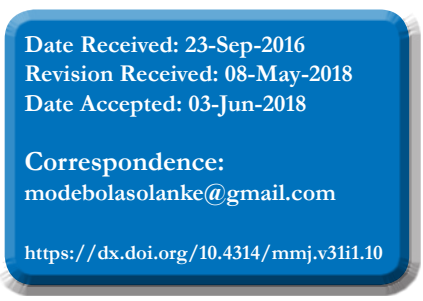

\begin{abstract}
\section{Background}

Most existing studies on unintended pregnancies tend to examine the influence of individual socio-demographic and health characteristics without sufficient attention to community characteristics. This study examines community characteristics influencing unintended pregnancies in Kenya.

Methods

Data were extracted from the 2014 Kenya Demographic and Health Survey (KDHS). The outcome variable was unintended pregnancy. The explanatory variables were selected individual and community level variables. The Multilevel mixed-effects logistic regression was applied. Results

Findings show $41.9 \%$ prevalence of unintended pregnancies. Community characteristics such as community education, community timing for initiation of childbearing, community fertility norms, and community media exposure significantly influence the likelihood of unintended pregnancies. The Intra-Cluster Correlation (ICC) provided evidence that community characteristics had effects on unintended pregnancies. Conclusion

There is evidence that community characteristics influence the prevalence of unintended pregnancies in Kenya. Community sensitisation and mobilisation should be central to all efforts aiming to reduce prevalence of unintended pregnancies.
\end{abstract}

Keywords: unintended pregnancy, pregnancy intention, women, community, Kenya

\section{Introduction}

Unintended pregnancies refer to pregnancies that occur at a time the woman would have preferred to wait till sometime in the future or when the woman no longer wants another child $^{1,2}$. Each year, across the world, there are about 85 million unintended pregnancies with developing countries accounting for 72.6 million of the total, and Eastern Africa accounting for the highest proportion in Africa ${ }^{3}$. At the prevailing level, unintended pregnancies remain an important global public health issue because of its adverse effects on the sexual and reproductive health of women, as well as adverse effects on children ${ }^{4-10}$. These effects include less likelihood of prolonged breastfeeding, induced abortions, maternal deaths, maternal disabilities and poor mental health, depression during pregnancy, neonatal deaths, and postpartum depression ${ }^{1,11-16}$. Against this backdrop, it is important to sustain research interest on the underlying factors associated with unintended pregnancies so as to inform policy and family planning programmatic decisionmaking.

However, most of the existing quantitative studies in Kenya and elsewhere ${ }^{17-22}$ tend to examine the influence of individual socio-demographic and health characteristics on unintended pregnancies without giving sufficient attention to community characteristics. Community characteristics are the distinct characteristics of the social group to which individuals belong. These characteristics may affect health outcomes either directly, for example unintended pregnancy, or indirectly by moderating how individuals' characteristics affect health outcomes. Although, individual characteristics may directly affect health outcomes, community characteristics represent a higher level of abstraction, and have independent effects on individual health outcomes such as unintended pregnancy after individual characteristics have been considered. The basic reason for such independent effects is that two individuals with similar individual characteristics may have different likelihood of a health outcome because they live in different communities. For instance, it is possible for a woman with very low education to reside in a community where most women are educated. The kind of social influence created by the fact that majority of women in the community are educated would differ from another context where majority in the community have no formal education. In the same vein, neither can it be argued that community characteristics 
are superior to individual characteristics nor can the reverse be substantiated. The empirical reality is that both types of characteristics help in providing a deeper understanding about different factors affecting an outcome. Hence, addressing the current prevalence of unintended pregnancies in Kenya may require not only the analysis of individual characteristics, but also the community characteristics. The need to give primacy to relevant community and social contexts in understanding health behaviour and outcomes is well established in literature ${ }^{23-24}$. Though a number of studies have examined community contexts of pregnancyrelated issues ${ }^{25-26}$, fewer studies ${ }^{27-28}$ have specifically investigated the influence of community characteristics on unintended pregnancies. The few existing studies mainly focused on community education, community poverty level, and geographic region. Other community variables such as community media exposure, community fertility norms and community level of teenage motherhood have rarely been linked to unintended pregnancies. This knowledge gap has been addressed in this study.

Two reasons account for selecting Kenya as a study location. Firstly, the current level of unintended pregnancies in Kenya is over 40 percent in spite of modest achievement of the family planning programme in the country ${ }^{29-30}$. This exerts enormous pressure on fertility and family planning programmes in the country, and may slow down fertility transition. Secondly, most existing studies on correlates of unintended pregnancies in Kenya ${ }^{31-32}$ did not analyse community characteristics in their investigations. The objective of the paper is, therefore, to examine community characteristics influencing unintended pregnancies in Kenya. The study was guided by the research question: Do community characteristics influence unintended pregnancies in Kenya?

\section{Methods \\ Study setting}

The Republic of Kenya is the third most populous country in Eastern Africa with an estimated 2015 population of 44.3 million persons ${ }^{33}$. The country's population grows mainly through natural increase. The current contraceptive prevalence rate for modern methods in Kenya is 53\% which is above the Eastern Africa's average of $35 \% \%^{33}$. Based on current Human Development Index, Kenya is rated as a low human development country with moderate inequality in gender, education and income ${ }^{34}$. Since 1967, the government of Kenya has implemented the national family planning programme as an integral part of the overall national development strategies, making Kenya one of the sub-Saharan African countries with the earliest national family planning programme ${ }^{35}$. With over fifty years of implementation, the family planning programme in Kenya has resulted in fertility decline and reduction in adverse reproductive health outcomes such as childhood mortality rate, maternal mortality, and HIV prevalence in the country $^{36}$. Although, the first National Reproductive Health Policy in Kenya was adopted only in $2007^{37}$, the country has appropriate policies and programmes to promote the sexual and reproductive health of the population.
These include the 2015 National Adolescent Sexual and Reproductive Health Policy, Kenya Health Policy (20122030), 2014 National Gender-Based Violence Policy, and the 2012 Population Policy for National Development ${ }^{38}$. However, in spite of the numerouspopulation and health policies, the sexual and reproductive health of men and women in Kenya remains poor with high prevalence of unintended pregnancies and clandestine abortion ${ }^{30}$. It was noted in the policy that lack of political commitment, poverty and a number of socio-cultural factors such as lack of female autonomy in decision-making, myths and misconceptions such as associating contraceptives with being promiscuous or health challenges, and negative attitudes hindered the progress of previous population and health programmes in the country ${ }^{35}$. Current efforts to improve sexual and reproductive health, particularly contraceptive prevalence in the country, include expansion of family planning service delivery points including community-based distribution, promotion of male involvement, integration of family planning with HIV/AIDS and other reproductive health services $^{35}$.

\section{Data source and sample design}

Data analysed in the study were extracted from the women's data of the 2014 Kenya Demographic and Health Survey (KDHS). The 2014 KDHS was implemented by national agencies led by the Kenya National Bureau of Statistics. The sample included in the survey was drawn from 39,679 households in 1,612 clusters (primary sampling unit) with 995 clusters in rural areas and 617 urban areas. A two-staged sampling technique was adopted for the survey. Detail design of the 2014 KDHS has been published ${ }^{36}$. The study did not analyse information from all the women covered in the survey. Analysis was restricted to women who were currently pregnant or whose last child was born within the last five years preceding the survey. The weighted sample size was 6,871 women. Data were requested from MEASURE DHS through online data access facility. Authorisation to analyse the dataset was granted by the organisation.

\section{Outcome variable}

The outcome variable was pregnancy intention categorised into intended and unintended. This was generated from information on the last child born during the last five years and from current pregnancy. The pregnancy intention of women who reported that they wanted their last child or current pregnancy were categorised as 'intended' while the pregnancy intention of those who reported that they wanted the child or pregnancy later and those who do not want the child or current pregnancy were categorised as 'unintended'.

\section{Explanatory and control variables}

The explanatory variables were five individual-level variables and five community-level variables. Five individual-level variables were analysed, namely: individual education, age at first marriage, current marital status, parity and employment status. These variables were selected on the basis of their significance in previous studies ${ }^{34,36,22}$. Age at first marriage was categorised into four groups, namely, 14 years or less, 15-19 years, 20-24 years and 25 years or older. Current 
marital status was grouped into two, namely: not currently married and currently married. Parity was measured by number of children ever born and was divided into three groups, namely: low parity (two or fewer children ever born), multiparity (three to four children ever born), and grand multiparity (five or more children ever born). Employment status was categorised as employed or unemployed.

The five community-level variables were community media exposure, community fertility norm, community education, community type of residence, and community level of teenage motherhood. Two of these variables (education and type of residence) have been analysed in an earlier study ${ }^{26}$. Community media exposure was generated from the combined frequencies of reading newspaper, listening to radio, and watching television within a week. Community fertility norm was generated from combination of individual ideal family size, while community initiation of childbearing was generated from combination of individual age at first birth. Community education was derived from combination of individual woman education.

To derive community variables, the method of aggregation was adopted by first setting a benchmark to indicate proportion of women in the community having the attribute or characteristic of interest and then aggregating the variable at the cluster (community) level. The proportions were then ranked and divided into three groups (tertiles). For instance, to derive community level of teenage motherhood, age at first birth was benchmarked at 18 years. All women who became mothers below age 18 years were combined and sorted by cluster to show the proportion of women who became mothers early. This proportion was then divided into three to indicate low, medium, or high proportions of teenage motherhood in the community. Previous studies that have explored community contexts using the DHS data have used similar method to derive community variables ${ }^{41-42}$.

Four household-level variables were selected for statistical control. These are women's autonomy, household wealth, spousal violence, and type of marriage. All the variables have shown significant influence on unintended pregnancies in previous studies ${ }^{40,8}$. Women's autonomy was based on responses to questions on participation in three household decisions, namely: decision on own health, purchase of large household items and visits to friends and relatives. Women who took all the decisions solely were defined as having 'high' autonomy, those who took part in the decision jointly with the male partner were defined as having 'partial' autonomy, and other women such as those who did not take part at all in the decisions were defined as having 'no' autonomy. Women were grouped as 'polygamous' or 'monogamous' if the partner had at least one other wife or not. The three types of spousal violence measured in the DHS (physical, sexual and emotional) were combined to form a single variable showing whether women had ever or never experienced at least one type of spousal violence.

\section{Statistical analyses}

All statistical analyses were performed using Stata 12.
Frequency distributions were used to describe sample characteristics and prevalence of unintended pregnancies. Simple cross tabulation of explanatory variables and pregnancy intention, and unadjusted binary logit coefficient with $95 \%$ confidence interval were used to examine the relationship between the study variables. Positive coefficient indicates positive relationship and negative coefficient indicate otherwise. The multilevel mixed-effects logistic regression analysis was applied. This method was selected for the study because of the hierarchical nature of the data ${ }^{43}$.

Model Specification:

$\log \left[\frac{\pi_{i j}}{1-\pi_{j}}\right]=\beta_{0}+\beta_{1} X_{1 j}+\ldots \beta_{n} X_{n i j}+u_{0 j}+e_{j}$

Where:

$\pi_{i j}$ is the log of odds of unintended pregnancy

$\left(1-\pi_{i j}\right)$ is the log of intended pregnancy

$\beta_{0}$ is $\log$ of the intercept

$\beta_{1} \ldots \beta_{n}$ are the regression coefficients

$X_{1 j} \ldots X_{n i j}$ are the individual and community characteristics included in the model $u_{0 j}$ are the random errors at cluster level $e_{i j}$ is the error term.

\section{Model Estimation}

The Stata $x$ tmelogit command was used to estimate the model parameters ${ }^{44}$. Five different models were fitted. Model 0 is the empty model which did not include any explanatory variable. Model 1 included only individual-level variables while Model 2 was based on the community-level variables. In Model 3, the individual and community variables were combined. The full model (Model 4) included all explanatory and control variables. The odds ratios with $95 \%$ confidence intervals were used to measure the fixed-effects (measure of association between the explanatory and outcome variables). The Intra-Class Correlation (ICC) was used to measure the random effects (measure of variation).

The ICC was calculated as:

$$
\frac{\sigma_{u}^{2}}{\sigma_{u}^{2}+\frac{\pi^{2}}{3}}
$$

where $\sigma_{u}^{2}$ is the variance at the community level and $\pi^{2} / 3$ is equal to $3.24^{43}$. The ICC expressed in percentage showed the variation in unintended pregnancies due to community characteristics.

\section{Model Diagnosis}

Model adequacy was examined by the Log-likelihood Ratio test (LR test). The LR test compares the fitted model with one-level ordinary linear regression. The result will indicate if the fitted model is adequate for the data being analysed. The Variance Inflation Factor (VIF) with mean VIF of 3.68 confirmed the absence of significant multicollinearity 
among the explanatory variables. The statistical significance for all tests was set at $\mathrm{p}<0.05$.

\section{Results}

Table 1 presents the socio-demographic profile of respondents. Educational attainments among the women showed dominance of primary education (54.6\%). Higher education was the least educational level attained among more than one-tenth $(13.3 \%)$, while majority of them had not witnessed any form of spousal violence. More than onethird of respondents $(39.6 \%)$ had high individual autonomy, but the proportion of respondents with moderate individual autonomy was nearly one-third of respondents (32.5\%). Household wealth was nearly evenly distributed among the women. However, the highest proportion of the women

Table 1: Respondents' socio-demographic characteristics and pregnancy intention, Kenya, selected women, 2014

\begin{tabular}{|c|c|c|c|c|c|}
\hline Characteristic & Number of Women & Percentage & \multicolumn{3}{|l|}{$\begin{array}{l}\text { Characteristic } \\
\text { Individual autonomy }\end{array}$} \\
\hline Education & 672 & 98 & Tow & 1920 & 279 \\
\hline None & & & & & 27.0 \\
\hline & 3,755 & 54.6 & Moderate & 2,231 & 32.5 \\
\hline Primary & 1,830 & 26.6 & High & 2,720 & 39.6 \\
\hline $\begin{array}{l}\text { Secondary } \\
\text { Higher }\end{array}$ & 614 & 9.0 & \multicolumn{3}{|l|}{ Type of marriage } \\
\hline Employment status & & & Monogamy & 4,860 & 70.7 \\
\hline Unemploved & 2,375 & 34.6 & Polygamy & 2,011 & 29.3 \\
\hline Emploved & 4,496 & 65.4 & \multicolumn{3}{|c|}{ Community education (proportion with at least secondary education) } \\
\hline Parity & & & Low & 1,690 & 24.6 \\
\hline Farily & 3,210 & 46.7 & Medium & 2,323 & 33.8 \\
\hline $\begin{array}{l}\text { Low ( } 2 \text { or fewer) } \\
\text { Multiparity (3-4) }\end{array}$ & 2,052 & 29.9 & High & 2,858 & 41.6 \\
\hline Grand multiparity ( 5 or more) & 1,609 & 23.4 & \multicolumn{3}{|c|}{ Community level of teenage pregnancy } \\
\hline Marital status & & & Low & 2,622 & 38.2 \\
\hline Mallial staus & 1,290 & 18.8 & Medium & 2,242 & 32.6 \\
\hline $\begin{array}{l}\text { Not currently married } \\
\text { Currently married }\end{array}$ & 5,581 & 81.2 & High & 2,007 & 29.2 \\
\hline Ace at first marriage & & & \multicolumn{3}{|c|}{ Fertility norm (proportion of large family size) } \\
\hline Age at iाrst marrlage & 1,049 & 15.3 & Low & 3,042 & 44.3 \\
\hline 14 years or less & 3,197 & 46.5 & Medium & 2,381 & 34.6 \\
\hline 15-19 years & 2,088 & 30.4 & High & 1,419 & 21.1 \\
\hline 25 vears or older & 537 & 7.8 & \multicolumn{3}{|c|}{ Community media exposure (proportion with access to mass media) } \\
\hline 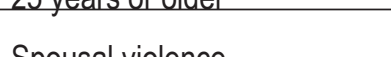 & & & Low & 1,762 & 25.6 \\
\hline spousal violence & 916 & 13.3 & Medium & 2,228 & 32.4 \\
\hline $\begin{array}{l}\text { Ever experlenced } \\
\text { Never experienced }\end{array}$ & 5,955 & 86.7 & High & 2,881 & 42.0 \\
\hline Houghald & & & \multicolumn{3}{|c|}{ Community residence type } \\
\hline $\begin{array}{l}\text { Household wealth quintile } \\
\text { Poorest }\end{array}$ & 1,379 & 20.0 & Urban & 2,676 & 38.9 \\
\hline
\end{tabular}

respondents. Nearly two-thirds of respondents were currently working. Low parous women were dominant among respondents. However, the proportion of multiparous women was nearly one-third of the respondents $(29.9 \%)$. Majority of the women were currently married. Nearly half of respondents became mothers within the 1519 years age interval, while nearly one-third of respondents (30.4\%) became mothers within the $20-24$ years age interval. However, more than one-tenth of respondents became mothers before reaching the lower limit of the conventional reproductive age. The proportion of women who had ever experienced at least one type of spousal violence was slightly belongs to households in the richest wealth quintile. Majority of respondents $(70.7 \%)$ were in monogamous unions. The prevalence of unintended pregnancies was more than onethird $(41.9 \%)$ among respondents.

Table 2 presents the relationship between explanatory and outcome variable. Education had mixed relationship with pregnancy intention. The proportion of unintended pregnancies was highest among women who had primary education $(48.3 \%)$, but the proportion reduced as educational level improved to secondary $(41.0 \%)$ and higher level $(23.7 \%)$. Only higher education was negatively 
Table 2: Bivariate relationship between socio-demographic characteristics and pregnancy intention

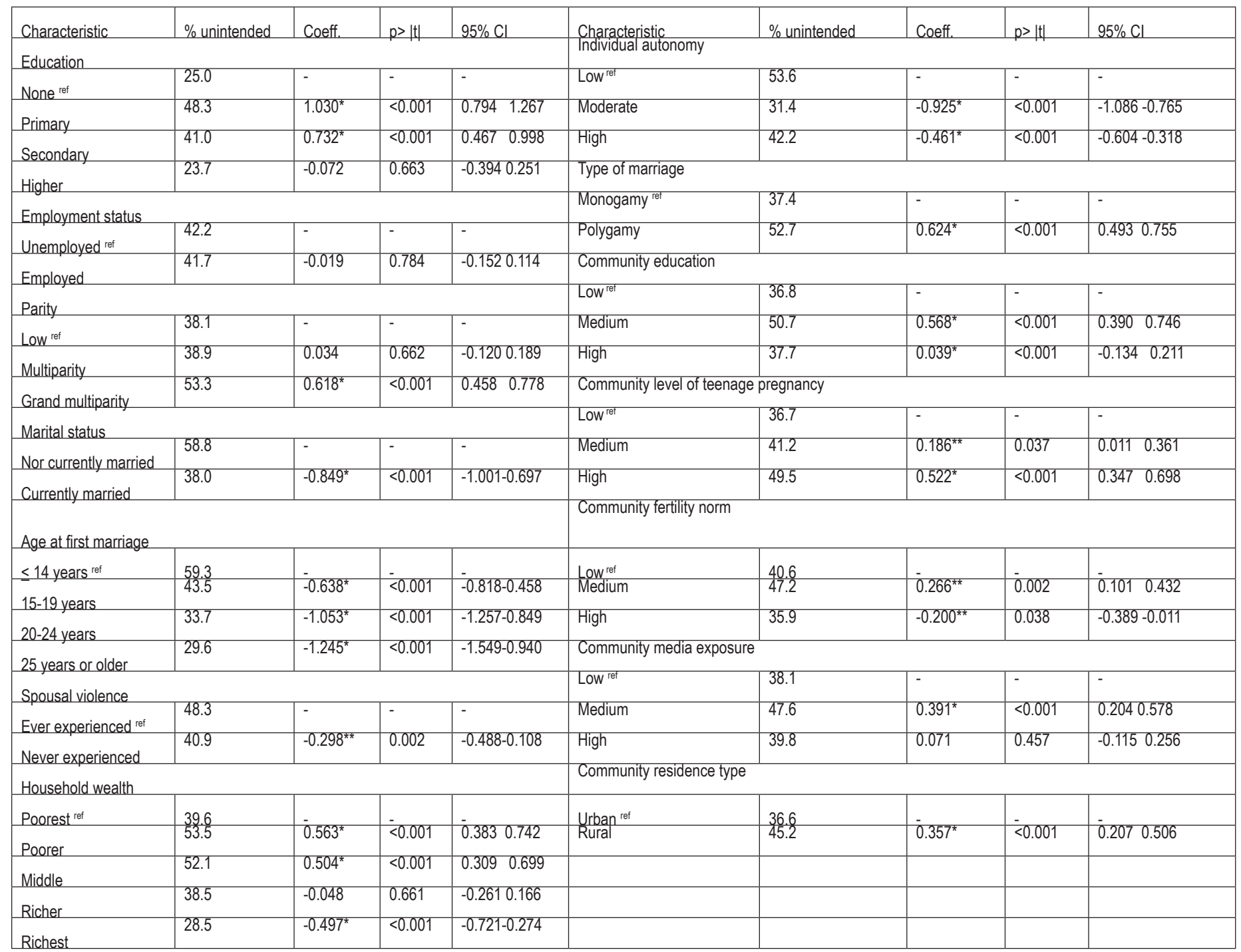

associated with pregnancy intention. Employment status and pregnancy intention were negatively associated with slightly higher proportion of unintended pregnancy among women who were not working compared with employed women $(42.2 \%$ vs. $41.7 \%)$. Parity and pregnancy intention were positively related with consistent increase in the proportions of unintended pregnancies as parity level increases. The relationship between marital status and pregnancy intention was negative. Women who were not currently married had higher proportion of unintended pregnancies compared with those currently married (58.8\% vs. $38.0 \%)$. Likewise, age at first marriage and pregnancy intention were negatively associated with higher proportions of unintended pregnancies among women who married at younger ages.

Spousal violence showed negative association with pregnancy intention with lower proportion of unintended pregnancies among women who had never experienced any type of spousal violence. Women's autonomy was negatively associated with pregnancy intention. The proportion of unintended pregnancies was highest among women with low autonomy and lowest among women with moderate autonomy, but women who had high autonomy had higher proportion of unintended pregnancies compared with women with moderate autonomy. Household wealth had mixed relationship with pregnancy intention. At the lower levels of the wealth groups, the relationship was positive, but negative at higher levels of the wealth groups. Type of marriage and pregnancy intention were positively associated with higher proportion of unintended pregnancies among polygamous women. With the exclusion of community fertility norm, all the community-level variables were positively associated with pregnancy intention.

Table 3 presents results of the fixed effects of the multilevel logistic regression. In Model 1, all individual characteristics, excluding employment status, significantly influenced the likelihood of unintended pregnancies. In Model 2, all the community characteristics exerted significant influence on the likelihood of unintended pregnancies. When the individual and community characteristics were combined in Model 3, there was no change in the pattern of influence on the likelihood of unintended pregnancies. However, in the full model (Model 4), there were slight changes in the pattern of influence on unintended pregnancies. All the individual characteristics revealed significant influence on unintended 
Table 3: Multilevel logistic regression showing fixed effects on unintended pregnancies

\begin{tabular}{|c|c|c|c|c|c|c|c|c|c|c|c|c|}
\hline \multirow[b]{2}{*}{ Characteristic } & \multirow{2}{*}{\multicolumn{3}{|c|}{ Mode 1}} & \multirow{2}{*}{$\begin{array}{l}\text { Model } 2 \\
\text { OR }\end{array}$} & \multicolumn{2}{|c|}{$x^{2}$} & \multirow{2}{*}{$\begin{array}{l}\text { Model } 3 \\
\text { OR }\end{array}$} & \multirow[b]{2}{*}{$p>|z|$} & \multirow[b]{2}{*}{$95 \% \mathrm{Cl}$} & \multirow{2}{*}{ Model 4} & \multirow{2}{*}{$p>|z|$} & \multirow[b]{2}{*}{$95 \% \mathrm{Cl}$} \\
\hline & & & $95 \% \mathrm{Cl}$ & & $p>|z|$ & $95 \% \mathrm{Cl}$ & & & & & & \\
\hline \multicolumn{13}{|l|}{ Education } \\
\hline None ref & - & - & - & & & & - & - & - & - & - & - \\
\hline Primary & $4.794^{*}$ & $<0.001$ & 3.6026 .379 & & & & $2.862^{*}$ & $<0.001$ & 2.2073 .709 & $2.845^{*}$ & $<0.001$ & 2.1923 .693 \\
\hline & $4.502^{*}$ & $<0.001$ & 3.3356 .078 & & & & $2.574^{*}$ & $<0.001$ & 1.9213 .449 & $2.816^{*}$ & $<0.001$ & 2.0823 .810 \\
\hline $\begin{array}{l}\text { Secondary } \\
\text { Higher }\end{array}$ & $2.503^{*}$ & $<0.001$ & 1.7873 .506 & & & & $1.558^{* *}$ & 0.013 & 1.0982 .212 & $2.081^{*}$ & $<0.001$ & 1.4423 .002 \\
\hline \multicolumn{13}{|c|}{ Employment status } \\
\hline Unemployed & - & - & - & & & & - & - & - & - & - & - \\
\hline Employed & 0.971 & 0.658 & 0.8541 .105 & & & & 0.893 & 0.088 & 0.7841 .017 & $0.859^{* *}$ & 0.023 & 0.7530 .980 \\
\hline \multicolumn{13}{|l|}{ Parity } \\
\hline Low ref & - & - & - & & & & - & - & - & - & - & - \\
\hline Multinarity & $1.369^{*}$ & $<0.001$ & 1.1771 .593 & & & & $1.364^{*}$ & $<0.001$ & 1.1731 .587 & $1.323^{*}$ & $<0.001$ & 1.1391 .536 \\
\hline $\begin{array}{l}\text { Grand } \\
\text { multiparity }\end{array}$ & $2.305^{*}$ & $<0.001$ & 1.9042 .792 & & & & $2.378^{*}$ & $<0.001$ & 1.9552 .894 & $2.220^{*}$ & $<0.001$ & 1.8342 .687 \\
\hline \multicolumn{13}{|l|}{ Marital status } \\
\hline$\underset{\text { ref }}{\text { Not c/married }}$ & - & - & - & & & & - & - & - & - & - & - \\
\hline $\begin{array}{l}\text { Currently } \\
\text { married }\end{array}$ & $0.413^{*}$ & $<0.001$ & 0.3340 .510 & & & & $0.424^{*}$ & $<0.001$ & 0.3440 .523 & $0.637^{* *}$ & 0.003 & 0.4710 .861 \\
\hline \multicolumn{13}{|c|}{ Age at first marriage } \\
\hline$\leq 14$ years $^{\text {ref }}$ & - & - & - & & & & - & - & - & - & - & - \\
\hline $15-19$ years & $0.617^{*}$ & $<0.001$ & 0.5080 .748 & & & & $0.616^{*}$ & $<0.001$ & 0.5080 .748 & $0.615^{*}$ & $<0.001$ & 0.5070 .747 \\
\hline 20 -24 vears & $0.487^{*}$ & $<0.001$ & 0.3910 .607 & & & & $0.496^{*}$ & $<0.001$ & 0.3970 .619 & $0.506^{*}$ & $<0.001$ & 0.4060 .630 \\
\hline $\begin{array}{l}25 \text { years or } \\
\text { older }\end{array}$ & $0.469^{*}$ & $<0.001$ & 0.3460 .636 & & & & $0.483^{*}$ & $<0.001$ & 0.3560 .655 & $0.513^{*}$ & $<0.001$ & 0.3790 .694 \\
\hline \multicolumn{13}{|c|}{ Community education } \\
\hline Low & & & & - & - & 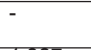 & - & - & - & - & - & - \\
\hline Medium & & & & $1.612^{*}$ & $<0.001$ & $\begin{array}{l}1.327 \\
1.958\end{array}$ & $1.461^{*}$ & $<0.001$ & 1.2061 .771 & $1.392^{*}$ & $<0.001$ & 1.1531 .680 \\
\hline High & & & & 1.068 & 0.529 & $\begin{array}{l}0.870 \\
1.312\end{array}$ & 1.133 & 0.264 & 0.9101 .412 & 1.171 & 0.157 & 0.411 .459 \\
\hline \multicolumn{13}{|c|}{ Community level of teenage pregnancy } \\
\hline Low & & & & - & - & $\overline{7}$ & - & - & - & - & - & - \\
\hline Medium & & & & 1.144 & 0.116 & $\begin{array}{l}0.967 \\
1.354\end{array}$ & 1.071 & 0.412 & 0.9091 .261 & 1.051 & 0.542 & 0.8951 .235 \\
\hline High & & & & $1.818^{*}$ & $<0.001$ & $\begin{array}{l}1.504 \\
2.197\end{array}$ & 1.482 & $<0.001$ & 1.2371 .775 & $1.403^{*}$ & $<0.001$ & 1.1771 .673 \\
\hline
\end{tabular}

pregnancies. For instance, women who attained higher education were twice likely to have unintended pregnancies compared with uneducated women $(\mathrm{OR}=2.081$; CI: 1.442 3.002); women who were working were $14.1 \%$ less likely to have unintended pregnancies (OR=0.859; CI: 0.753-0.980); and grand multiparous women were more than twice likely to have unintended pregnancies compared with low parity women $(\mathrm{OR}=2.220$; CI: 1.834-2.687).

Table 4 presents results of the random effects of the multilevel logistic regression. The LR test confirms that all the fitted models were adequate for the data analysed. In the empty model, the ICC showed that the community- level variables accounted for $21.4 \%$ of the variation in unintended pregnancies among women in Kenya. In Model 1 , the community-level variables accounted for $12.1 \%$ of the variation, while in Model 2, community-level variables accounted for $13.3 \%$ of the variation in unintended pregnancies among women. In Model 3, it accounted for $10.0 \%$, while in Model 4, it accounted for $8.9 \%$ of the variation in unintended pregnancies. These results confirm that community characteristics do have effects on the prevalence of unintended pregnancies among women in Kenya. 
Table 3 continued: Multilevel logistic regression showing fixed effects on unintended pregnancies

\begin{tabular}{|c|c|c|c|c|c|c|c|c|c|c|c|}
\hline Characteristic & \multicolumn{2}{|l|}{\begin{tabular}{|l|} 
Model 1 \\
$R$
\end{tabular}} & Model 2 & $|p>| z \mid$ & $95 \% \mathrm{Cl}$ & $\begin{array}{l}\text { Model } 3 \\
R\end{array}$ & $p>z$ & $95 \% \mathrm{Cl}$ & $\begin{array}{l}\text { Model } 4 \\
\text { OR }\end{array}$ & $|p>| z \mid$ & $95 \% \mathrm{Cl}$ \\
\hline \multicolumn{12}{|c|}{ Community fertility norm } \\
\hline Jow ref & & & -- & - & - & - & - & - & - & - & - \\
\hline Low & & & 1.080 & 0.371 & 0.9131 .277 & 1.011 & 0.896 & 0.8591 .189 & 0.987 & 0.870 & 0.8401 .159 \\
\hline Hedium & & & $0.452^{*}$ & $<0.001$ & 0.3610 .565 & 0.517 & $<0.001$ & 0.4130 .648 & $0.529^{*}$ & $<0.001$ & 0.4230 .662 \\
\hline \multicolumn{12}{|c|}{ Community media exposure } \\
\hline \multicolumn{12}{|c|}{\begin{tabular}{|l|l} 
Low ref & \\
\end{tabular}} \\
\hline & & & $1.606^{*}$ & $<0.001$ & 1.3191 .955 & $1.422^{*}$ & $<0.001$ & 1.1761 .719 & $1.432^{*}$ & $<0.001$ & 1.1861 .728 \\
\hline Medium & & & $1.380^{* *}$ & 0.002 & 1.1281 .689 & $1.303^{*}$ & $<0.001$ & 1.0701 .586 & $1.468^{*}$ & $<0.001$ & 1.1991 .797 \\
\hline \multicolumn{12}{|c|}{ Community residence type } \\
\hline Urban ref & & & -- & - & - & - & - & - & - & - & - \\
\hline Ulodil & & & $1.534^{*}$ & $<0.001$ & 1.3031 .806 & $1.355^{*}$ & $<0.001$ & 1.1591 .584 & 1.088 & 0.293 & 0.9291 .275 \\
\hline \multicolumn{12}{|c|}{ Spousal violence } \\
\hline Fyer ref & & & & & & & & & - & - & - \\
\hline Lid & & & & & & & & & $0.760^{* \pi}$ & 0.002 & 0.5390 .905 \\
\hline Never & & & & & & & & & & & \\
\hline \multicolumn{12}{|c|}{ Women autonomy } \\
\hline Low ref & & & & & & & & & - & - & - \\
\hline Moderate & & & & & & & & & $0.643^{*}$ & $<0.001$ & 0.5130 .806 \\
\hline High & & & & & & & & & 0.964 & 0.732 & 0.7791 .191 \\
\hline \multicolumn{12}{|c|}{ Household wealth } \\
\hline \multicolumn{12}{|l|}{ Poorest ref } \\
\hline Doner & & & & & & & & & $1.224^{* *}$ & 0.041 & 1.0091 .486 \\
\hline Poorer & & & & & & & & & 1.219 & 0.059 & 0.9921 .497 \\
\hline Middle & & & & & & & & & $0.751^{\text {** }}$ & 0.013 & 0.6000 .941 \\
\hline Richer & & & & & & & & & $0,510^{*}$ & $<0001$ & 03840677 \\
\hline Richest & & & & & & & & & 0.510 & $<0.001$ & $0.3840 .67 \pi$ \\
\hline \multicolumn{12}{|c|}{ Type of marriage } \\
\hline Monogamv ref & & & & & & & & & - & - & - \\
\hline Polvarmy & & & & & & & & & $1.275^{* *}$ & 0.014 & 1.0501 .547 \\
\hline
\end{tabular}

Table 4: Multilevel logistic regression showing random effects on unintended pregnancies

\begin{tabular}{|c|c|c|c|c|c|c|c|c|c|c|c|c|c|c|c|}
\hline & \multicolumn{3}{|c|}{ Empty Model } & \multicolumn{3}{|c|}{ Model1 } & \multicolumn{3}{|c|}{ Model2 } & \multicolumn{3}{|c|}{ Model 3} & \multicolumn{3}{|c|}{ Model 4} \\
\hline Parameter & Est. & S. Err. & $95 \% \mathrm{Cl}$ & Est. & S. Err. & $95 \% \mathrm{Cl}$ & Est. & S. Err. & $95 \% \mathrm{Cl}$ & Est. & S. Err. & $95 \% \mathrm{Cl}$ & Est. & S. Err. & $95 \% \mathrm{Cl}$ \\
\hline $\begin{array}{l}\text { Variance } \\
\text { (Community) }\end{array}$ & 0.897 & 0.148 & $.65-1.24$ & 0.453 & 0.096 & $.23-68$ & 0.503 & 0.096 & $.34-73$ & 0.365 & 0.083 & $.23-57$ & 0.322 & 0.077 & $.20-.51$ \\
\hline Variance (Individual) & 0.457 & 0.383 & $.09-2.34$ & 0.462 & 0.418 & $.08-2.72$ & 0.478 & 0.379 & $.01-2.26$ & 0.454 & 0.419 & $07-2.77$ & 0.412 & 0.405 & $.06-2.83$ \\
\hline $1 \mathrm{CC}(\%)$ & \multicolumn{3}{|l|}{$21.4 \%$} & \multicolumn{3}{|l|}{$12.1 \%$} & \multicolumn{3}{|l|}{$13.3 \%$} & \multicolumn{3}{|l|}{$10.0 \%$} & \multicolumn{3}{|l|}{$8.9 \%$} \\
\hline LR test & \multicolumn{3}{|c|}{$L R \times 2=236.6 ; 0<0.001$} & \multicolumn{3}{|c|}{ LR $\times 2=70.6 ; 0<0.001$} & \multicolumn{3}{|c|}{$L R \times 2=236.6 ; 0<0.001$} & \multicolumn{3}{|c|}{$\operatorname{LR} \times 2=236.6 ; 0<0.001$} & \multicolumn{3}{|c|}{$L R \times 2=236.6 ; 0<0.001$} \\
\hline
\end{tabular}

\section{Discussion}

This study examined community characteristics affecting unintended pregnancies in Kenya. The multilevel analyses carried out in the study were unique when compared to previous studies in Kenya because it estimated extent of variations in unintended pregnancies attributable to factors other than individual socio-demographic characteristics. These were missed out in previous studies ${ }^{22,30,31}$. The analysis further demonstrates the fact that pregnancy intention is influenced at multiple levels, which suggests that interventions to reduce unintended pregnancies may achieve more results if they are refocused to give due attention to all levels of influence.

The significance of individual socio-demographic characteristics such as parity, type of marriage, and marital status were upheld in the study in line with findings in previous studies ${ }^{19}, 20,32$ indicating the appropriateness of strategies that target the variables in the current population policy. But contrary to findings in previous studies ${ }^{19,20,32}$, the study found higher prevalence of unintended pregnancies among educated women. This is possible if the proportion of women reporting unintended pregnancies consists of substantial number of young and unmarried women. An evolving trend already reported in Kenya is the practice of 
young girls and women with reasonable education to cohabit with male partners without formal marriage $e^{30}$. This could give rise to high number of unintended pregnancies among educated women. It is therefore important for programmes to maintain focus on young women particularly those in cohabiting relationships.

Two findings on the community characteristics have implications for policy in the study area. Firstly, the study confirms that the extent of unintended pregnancies in Kenya is high. The $41.9 \%$ prevalence found in the study is comparable to the $41 \%$ reported in a study conducted in urban slums of Kenya ${ }^{22}$, although lower than the $47 \%$ reported in another study in Kenya ${ }^{30}$. This result also buttressed findings that unintended pregnancies were high in East $\mathrm{Africa}^{3}$. The high prevalence in Kenya suggests that in spite of the country's near universal knowledge of modern contraceptives and modest achievements of the family planning programme since its inception in $1967^{35}$, more progress needs to be made with respect to utilisation of modern methods as contraceptive knowledge alone cannot address the need either to space pregnancy or limit childbearing.

Unintended pregnancies among women who know about modern contraceptive methods especially educated women, as found in the study, indicate an unmet need for family planning which has adverse effects on women's sexual and reproductive health. This could create more demand for unsafe abortions in the country. These were well noted in previous research efforts in the country ${ }^{30}$. The gap between contraceptive knowledge and use may be due to changes in socio-cultural norms of family formation and childbearing. With increasing rise in the proportions of young girls who are delaying marriage and childbearing as a result of education, exposure to sexual intercourse and unintended pregnancies may also be high as revealed in the bivariate analysis. The likelihood will increase if many young women accept cohabitation with male partners prior to formal marital union in the absence of effective contraceptive use.

It is therefore imperative that efforts be scaled up to address xisting unmet needs for family planning among women particularly in rural communities where more women live. The current policy in the country has already noted the need for community participation in contraceptive distribution ${ }^{35}$, but more measures could still be developed if community engagement is strengthened through mobilisation and sensitisation on health issues affecting women's health in the communities. For instance, it could be more strategic to allow community members identify how best to distribute and ensure utilisation of modern contraceptives among women in specific communities.

More importantly, contraceptive and family planning programmes for women should be integrated into community economic empowerment programmes because one misconception that usually hinders contraceptive utilisation particularly in rural communities is the belief that a contraceptive is solely for limiting childbearing. The economic and health motivation for family planning may not be clear to many individuals and families in rural communities. Current efforts in the country have only attempted to integrate family planning with HIV/AIDS and other reproductive health services; it is now time to explore the possibility of integrating family planning programmes into community economic empowerment programmes for women. This will serve two purposes. One, it may clear myths and misconception that family planning solely focuses on childbearing by providing information on the nondemographic or non-health benefits of family planning use. Two, it will help to link family planning to poverty reduction which is one of the factors affecting sexual and reproductive health in the country as noted by the existing population policy ${ }^{35}$.

Secondly and contrary to expectation, the study revealed that women in communities with high prevalence of large ideal family size norm had lower prevalence of unintended pregnancies, and women in communities with high media exposure and education had higher prevalence of unintended pregnancies. These results are subtle and needs careful interpretation. In communities with either large family size norm or communities with low media exposure, there is a likelihood of wrong perception of what constitute unintended pregnancies. In traditional communities, children are cultural imperatives and childbearing is the main role for being a wife ${ }^{2}$. In such settings, unintended pregnancies are likely to be grossly under-reported. This also accounts for why some women give non-numeric responses to survey questions on their ideal family size ${ }^{2}$. On the contrary, educated women and women with reasonable exposure to mass media are likely to understand the meaning of unintended pregnancies, and give more accurate report of its incidence or prevalence than women in traditional communities. This may explain higher prevalence in communities with higher education and exposure to mass media as found in the study. It is therefore not out of place to explore different indicators for measuring unintended pregnancy for women in modern and traditional settings. Alternatively, the government could partner media institutions in the country to promote more awareness about unintended pregnancies in traditional communities ${ }^{30}$.

In spite of the agreement of most of the findings with earlier findings, the analysis carried out has some drawbacks that may limit inferences made in the study. Though the analysis revealed that the extent of unintended pregnancies was high in the study area, nevertheless, the prevalence may still be an underestimate due to the possibility of some women reporting an unwanted pregnancy as intended during the survey. Besides, the retrospective reporting of pregnancy or fertility intention by some women may not be totally accurate due to challenges in recalling past events. Although findings from the study suggest that community-based family planning initiatives may reduce the prevalence of unintended pregnancies through improved access to family planning methods, the study is not able to identify priority communities for such intervention. This is due to the need to fulfil one of the conditions for which authorisation was granted to the use of the data, that is, ensuring that individuals and communities analysed in the study remain anonymous. Also, the study is not able to ascertain the extent of homogeneity in the communities analysed. The 
DHS data rarely provides information on the homogeneity or heterogeneity of surveyed communities. However, the existence of mixed populations in some communities may have implications for the significance of contextual effects.

\section{Conclusion}

The study confirmed the significance of individual characteristics such as marital status, parity, and spousal violence for understanding pregnancy intention. The study further provided evidence that community-level variables such as community level of teenage motherhood, community media exposure and community fertility norms had influence on the prevalence of unintended pregnancies in Kenya. Community sensitization and mobilization should be central to all efforts aiming to reduce prevalence of unintended pregnancies. The possibility of integrating family planning programme with community economic empowerment programme for women in the communities should be explored.

\section{Declarations}

Ethics approval and consent to participate. The study analysed secondary data. Authorisation was granted by MEASURE DHS. The KDHS was approved by the ICF International Review Board and National Ethic Committee, Kenya. Informed consent of participants was obtained prior to all interviews in the survey.

\section{Consent for publication}

Not Applicable

\section{Availability of data and materials}

The dataset supporting the conclusions of this article is available online at www.measuredhs.com.

\section{Competing interests}

The authors declare no competing interests.

\section{Funding}

Not applicable.

\section{Acknowledgements}

The authors appreciate and thank ICF International and MEASURE DHS Project for making the datasets available for use and for granting prompt authorisation to analyse the data.

\section{References}

1. Marston C, Cleland J. Do unintended pregnancies carried to term lead to adverse outcomes for mother and child? An assessment in five developing countries. Pop Stud-J Demog. 2003; 57(1): 77-93, doi: 10.1080/0032472032000061749

2. Vadnais D, Kols A, Abderrahim N. women's lives and experiences: Changes in the past ten years. Calverton, Maryland, USA: ORC Macro; 2006. Available from: http://www.dhsprogram.com/pubs/pdf/OD40/ OD40.pdf

3. Sedgh G, Singh S, Hussain R. intended and unintended pregnancies worldwide in 2012 and recent trends. Stud Fam Plann. 2014; 45(3): 301314. doi: $10.1111 /$ j.1728-4465.2014.00393.x

4. Gipson JD, Koenig MA, Hindin M. The effects of unintended pregnancy on health outcomes: a review of the literature. Stud Fam Plann. 2008; 39(1): 18-38.

5. Tsui AO, McDonald-Mosley R, Burke AE. Family Planning and the Burden of Unintended Pregnancies. Epidemiol Rev. 2010, 32(1): 152-174, doi: 10.1093/epirev/mxq012

6. Singh S, Sedgh G, Hussain R. Unintended pregnancy: Worldwide levels, trends, and outcomes. Stud Fam Plann. 2010; 41(4): 241-250.

7. Peipert JF, Madden T, Allsworth JE, Secura GM. Preventing unintended pregnancies by providing no-cost contraception. Obstet Gynecol. 2012; 120(6): 1291-1297. doi: http://10.1097/AOG.0b013e318273eb56.

8. Salazar M, San Sebastian M. Violence against women and unintended pregnancies in Nicaragua: A population-based multilevel study. BMC Womens Health. 2014; 14:26, doi: 10.1186/1472-6874-14-26

9. Eliason S, Baiden F, Yankey BA, Awusabo-Asare K. Determinants of unintended pregnancies in rural Ghana. BMC Pregnancy Childbirth. 2014; 14:261, doi: 10.1186/1471-2393-14-261

10. Dhakal S, Song JS, Shin DE, Lee TH, So AY, Nam EW. Unintended pregnancy and its correlates among currently pregnant women in the Kwango District, Democratic Republic of the Congo. Reprod Health. 2016; 13:74. doi: 10.1186/s12978-016-0195-z

11. Hromi-Fiedler AJ, Pe'rez-Escamilla R. Unintended pregnancies are associated with less likelihood of prolonged breast-feeding: an analysis of 18 Demographic and Health Surveys. Public Health Nutr. 2005; 9(3): 306-312, doi: 10.1079/PHN2005856

12. Karacam Z, Sen E, Amanak K. Effects of unplanned pregnancy on neonatal health in Turkey: A case-control study. Int J Nurs Pract. 2010; 16: 555-563, doi: 10.1111/j.1440-172X-2010.01881.x

13. Karacam Z, Onel K, Gercek E. Effects of unplanned pregnancy on maternal health in Turkey. Midwifery. 2011; 27(2): 288-293, doi: 10.1016/j. midw.2009.07.006

14. Singh A, Chalasani S, Koenig MA, Mahapatra B. The consequences of unintended births for maternal and child health in India. Pop Stud-J Demog. 2012; 66(3): 223-239, doi: 10.1080/00324728.2012.697568

15. Yanikkerem E, Ay S, Piro N. Planned and unplanned pregnancy: Effects on health practice and depression during pregnancy. J Obstet Gynaecol. 2013; 39(1): 180-187, doi:10.1111/j.1447-0756.2012.01958.x

16. Russo NF. Abortion, unwanted childbearing, and mental health. Salud Mental. 2014; 37(4): 283-291.

17. Gomez AM. Sexual violence as a predictor of unintended pregnancy, contraceptive use and unmet need among female youth in Columbia. J Womens Health (Larchmt). 2011; 20(9): 1349-56, doi: 10.1089/ jwh.2010.2518

18. Askew I. Causes and Consequences of Unintended Pregnancies in Developing Countries. Presentation at Meeting of International Committee for Contraceptive Research Pioneered Modern Long-Acting Methods of Contraception; 2012; Population Council, New York, USA, Available from: www.popcouncil.org/uploads/pdfs/events/2012ICCR Askew.pdf

19. Faye CM, Speizer IS, Fotso JC, Corroon M, Koumtingue D. Unintended pregnancy: magnitude and correlates in six urban sites in Senegal. Reprod Health. 2013; 10: 59, doi: 10.1186/1742-4755-10-59

20. Mallard SR, Houghton LA. Socio-demographic characteristics associated with unplanned pregnancy in New Zealand: implications for access to preconception healthcare. Aust N Z J Obstet Gynaecol. 2013; 53(5): 498-501. doi: 10.1111/ajo.12074

21. Fotso JC, Izugbara C, Saliku, T, Ochako R. Unintended pregnancy and subsequent use of modern contraceptive among slum and non-slum women in Nairobi, Kenya. BMC Pregnancy Childbirth. 2014; 14, 224, doi: 10.1186/1471-2393-14-224

22. Beguy D, Mumah J, Gottschalk L. unintended pregnancies among young women living in urban slums: Evidence from a prospective study in Nairobi City, Kenya. PLoS ONE. 2014; 9(7): e101034, doi:10.1371/ journal.pone.0101034 